\title{
Performance Analysis of Supply Chain Networks using Petri nets
}

\author{
N. R. Srinivasa Raghavan \\ Dept. of Management Studies \\ Indian Institute of Science, Bangalore, India 560012 \\ e-mail: raghavan@mgmt.iisc.ernet.in
}

\author{
N. Viswanadham \\ Dept. of Mechanical and Production Engg. \\ National Univ. of Singapore, Singapore 119260 \\ e-mail: mpenv@nus.edu.sg
}

\begin{abstract}
In this paper, we investigate dynamic modeling tcchniques for analyzing supply chain networks using generalized stochastic Petri nets (GSPN). The customer order arrival process is assumed to be Poisson and the service processes at the various facilities of the supply chain are assumed to be exponential. Our modeling method accounts for both the logistics process as well as the interface processes that exist between any two members of the supply chain. We compare two production planning and control policies, the make-tostock and the assemble-to-order systems and discuss their merits. Locating the decoupling point in the supply chain is a crucial decision. We formulate the problem as a total cost minimization problem with the total cost comprising the inventory carrying cost and delay costs. We use the framework of integrated GSPN-queueing network modeling, with the GSPN at the higher level and a generalized queueing network at the lower level.
\end{abstract}

\section{The Supply Chain Process: An Overview}

Manufacturing supply chain networks (SCNs) are formed when manufacturing and service providers such as the original equipment manufacturers, raw material and component vendors, logistics operators, warehouse managers, etc., enter into a strategic alliance with a view to deliver value to the customers. Modeling and analysis of such a complex network is crucial for performance evaluation and benchmarking.

Supply chain performance depends on all the constituents of the supply chain. Long term issues in SCP involve location of production and inventory facilities, choice of alliance partners such as the suppliers and distributors, and the logistics chain. The long term decisions also include choosing between make to order and make to stock policies, degree of vertical integration, capacity decisions of various plants, amount of flexibility in each subsystem, etc.

\subsection{Brief Literature Sırvey}

In this section, we bricliy survey various articles available on supply chains. The performance modeling of supply chain networks ( $\mathrm{SCN}$ ) follows the same methodology as other discrete event systems. Basically there are three an- alytical methods: Markov chains, queuing networks and stochastic Petri nets. Discrete event simulation is another often used tool, while mathematical programming techniques are used mostly for strategic decision making. The stochastic models generally deal with tactical and operational level decisions.

In [3], the authors consider global manufacturing and distribution networks and formulate mixed integer optimization programs. A comprehensive deterministic model for supply chain management is provided in [1]. Because of their large scale, such models are often difficult to solve to optimality. Authors in [2] describe reengineering of supply chain using queueing network models.

A comprehensive review of multi-echelon inventory control models can be found in [10]. In [5], the authors consider a supply network model to generate base stock levels at each store so as to minimize the overall inventory capital and guarantee the customer service requirements.

Development of systems dynamics and simulation models for understanding issues of supply chain decision making has gained importance in recent years $[4,6]$. Simulation models can provide for comprehensive supply chain modeling considering the strategic, tactical, and operational elements. But these models can only evaluate the effectiveness of a predefined policy.

\section{Specification of Supply Chains}

\subsection{Configuration}

This could be of four major types including serial (tandem), converging, diverging, and converging-diverging (or network) structures.

\subsection{Operational Issues}

Another important ingredient of the supply chain specification is the production planning and control(PPC) methodology. Any order for an end product triggers a series of work processes in the supply chain which have to be completed so that the end customer order is satisfied. The PPC specifies the control approach for the flow of information and material in the supply chain. This is in general of three broad categories: Make-to-stock, Make-to-order, and Assembleto-order, For a good overview of these policies, see [4]. 


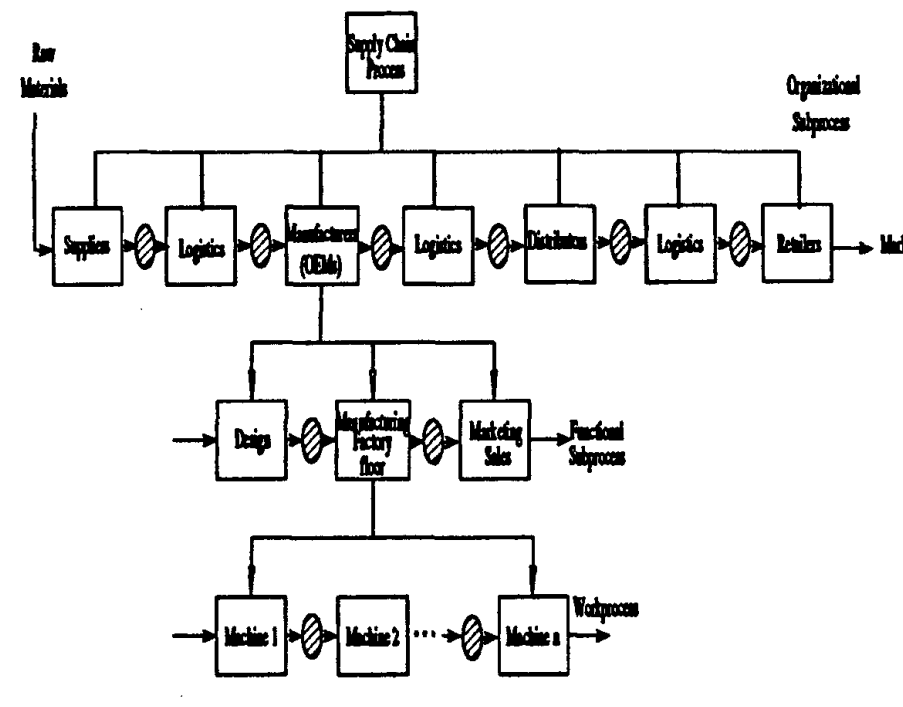

Figure 1: The supply chain process hierarchy

\section{Hierarchical Analysis of the Supply Chain Process}

For the purpose of modeling and analysis, we decompose the SCP as in Figure 1. As we said earlier, the SCP starts with procurement of raw materials and ends with supplying the material to the markets. The SCP transits several organizations and each time a transition is made, logistics is involved. Also since each of the organizations is under independent control, there are interfaces between organizations and material and information flow depend on how these interfaces are managed. We define interfaces as the procedures and vehicles for transporting information and materials across functions or organizations such as negotiations, approvals (so called paper work), decision making, and finally inspection of components/assemblies etc. For example, the interface between a supplier and manufacturer involves procurement decisions, price and delivery negotiations at the strategic level and the actual order processing and delivery at the operational level. The coordination of the SCN plays a big role in the over all functioning of the SCP. In most cases, there is an integrator for the network, who could be an original equipment manufacturer, who coordinates the flow of orders and materials through out the network. The forecasts and orders for each of the products manufactured by the supply chain are collected and converted into production and logistics schedules. They are then converted as component orders to the first tier suppliers using the bill of materials, who in turn will order for raw materials from second tier suppliers. The material then flows in the forward direction.

We assume that each of the organizations does its production scheduling in a decentrilized fashion. In modeling supply chains, two issues are veiy important. They include logistics and interfaces between crganizations.

Let us consider Figure $l$ again. Each of the organizations of the supply chain is by itself large scale and is governed by a functional organizational structure (we show this for the OEM in Figure 1). Each of the functions is again subdivided into work processes. We have shown a three layered hierarchy in the Figure 1 but in some supply chains there could be more layers. In the figure, we have prominently shown the interfaces, and logistics. The logistics take the form of inventory packaging, warehousing, transportation from one organization to another, etc.

The logistics could be supplier owned, buyer owned, or may be a third party operated one. To avoid inventories, stock-outs and production stoppages, it is important that the supplier, the buyer, and the logistics be properly coordinated. The coordination, also called the interface management, takes the form of strategic alliances, electronic partnership, vendor managed inventories, etc., which are agreements for a certain period of time. Interface management becomes more critical if international sourcing and marketing are involved.

\subsection{Performance Measures}

Performance measures are traditionally defined for an organization and are typically financial in nature, such as market share and return on sales or investment. This approach is fraught with many ills. First of all, financial indicators are lagging metrics that are a result of past decisions and are too old to be useful in operational performance improvement. Secondly, most companies do business with multiple partners selling multiple products, the individual financial statements do not delineate the winners and the losers. More importantly, several organizations are involved in the product manufacture and delivery to the customers, individual financial statements do not give a complete picture of the performance of the product or the process.

Recently there are efforts to define and determine nontraditional performance measures such as lead time, quality, reliable delivery, customer service, rapid product introduction, and flexible capacity. Time compression, quality improvement, product variety, and customer focus are the four pillars of modern business strategy. It is often argued that if these fundamental performance issues are managed well, then the cost reductions automatically follow.

\section{Mathematical Models}

Supply chains need to be modeled at various degrees of abstraction in order to arrive at strategic, tactical, or operational level decisions. For instance, it may not be required to incorporate daily data from shop floor when we are dealing with the supply chain location problem [7]. Similarly, when we need to schedule a particular facility of the supply chain, we need to include more details. In this paper, we intend to abstract the supply chain at the aggregate level, in particular, the organization level sub-processes of the supply 


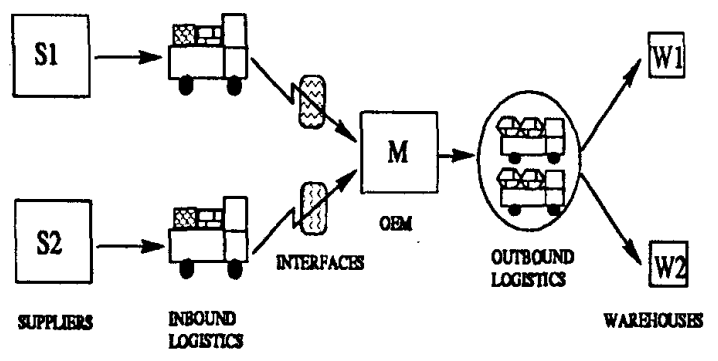

Figure 2: The supply chain considered for Petri net modeling

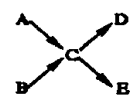

Figure 3: Product structure for supply chain considered

chain shown in Figure 1. The information required for such modeling, like the processing times at the various facilities, are assumed to be available $a$ priori. We wish to study the dynamics of the supply chain, especially, the impact of logistics and interfaces, and the manufacturing philosophies, on the performance measures that we define later. Such an aggregate level analysis of factory floors is common in literature [8]. We use the framework of generalized stochastic Petri nets (GSPN) for the analysis. See [9] for an excellent overview on GSPNs applied to manufacturing systems.

\section{Performance Analysis Illustrated}

Consider the supply chain network shown in Figure 2, with two suppliers for two sub-assemblies and a manufacturing plant for assembling these, as also, two separate vehicles to transport the sub-assemblies to the plants. There are two distribution centers acting as the end customers for the products. The inter arrival time of orders for the end products are assumed to be exponential in distribution. All processing and transporting activities are also exponentially distributed. We model this supply chain network under two different policies for material flow, viz., make-to-stock and assembleto-order.

The product structure considered for study is shown in Figure 3. There are two end products $D$ and $E$ which are available at warehouses $W_{1}$ and $W_{2}$ respectively. The demand for these two products are stochastic. The end products $D$ and $E$ are assembled at the final assembly plant or OEM, namely, $M$. The common base sub-assembly for $\mathrm{D}$ and $\mathrm{E}$ is C. Also, $C$ is assembled from raw materials $A$ and $B$ provided by suppliers $S_{1}$ and $S_{2}$ respectively. Inventories of $\mathrm{A}$, $B, C, D$, and $E$ are maintained at the respective facilities.

We model the dynamics of such a supply chain network, at a very aggregate level, by using Petri nets. Our modeling is more generic and wholesome than current approaches in the following sense. We consider the logistics process also in the study. Logistics is typically given a cursory treatment in available literature by assigning constant values for the transportation times. We allow for random logistics times. Also, crucial issues like interfaces shown in Figure 1, are not usually considered in current literature. We assume that the average times spent at the interface servers are known, along with their variances. Since we model such a network using GSPNs, we consider exponential service times only. Although more general distributions can be included, since our aim is to show how the dynamics of a supply chain can be captured by GSPNs, we limit our study to the exponential case. We note that while the inbound logistics into $M$ is managed by the suppliers individually, the outbound logistics out of $M$ is managed by the manufacturing plant itself. This is brought out in Figure 2 by having a common pool of logistics carriers that move products out of $M$.

We consider continuous review reorder point kind of inventory control policy. For ease of analysis, we assume that each arriving order for $\mathrm{D}$ or $\mathrm{E}$, is for $\mathrm{a}$ batch rather than single items. Since there is a join or assembling operation at $M$, the targeted inventories of A and B are integral multiples of C. With a similar argument, the targeted inventory of $C$ is an integral multiple of the sum of the targeted inventories of D and $E$. The latter are obtained by standard forecasting techniques, and are fixed, given parameters to our model. We hasten to add that our models can also accommodate the case wherein the stock piles are not integral multiples.

Stock piles of $D$ and $E$ are replenished at fixed reorder points, which are preset. The Petri net for the above case is shown in Figure 4. The description of the GSPN is given in Tables 1-2. We define enabling functions for the transitions $t A, t B, t C, t D$, and $t E$. These immediate transitions are enabled only when the tokens in the places representing inventory for $A, B, C, D$, and $E$ reach their reorder points, respectively. Observe that once this condition is satisfied, the transitions can keep on firing indefinitely. To avoid this, we define inhibitor arcs from places $P A^{\prime}, P B^{\prime}, P C^{\prime}, P D^{\prime}$, and $P E^{\prime}$, to the above transitions. Thus these places signify that material is already on order. The tokens from the above places are removed once the material is available for transporting to the respective inventory locations, which occurs when there is a token in the places $P 5, P 6, P 9, P 13$, and $P 14$. The initial marking (as shown in the GSPN) consists of tokens in places $P 7, P 8, P 10, P 16$, and $P 17$, equalling in number to the respective targeted finished goods inventory of $A, B, C, D$, and $E$.

We add an interesting dimension to the above model. Instead of making the final products D and E to stock, what would happen if they were assembled to order, from the common base component $C$ ? The make-to-stock kind of system offers better serviceability in terms of faster access to end products $\mathrm{D}$ and $\mathrm{E}$, thus reducing the probability of back ordering them. This naturally implies holding excess finished goods inventory which may get obsolete, if customer demands are 


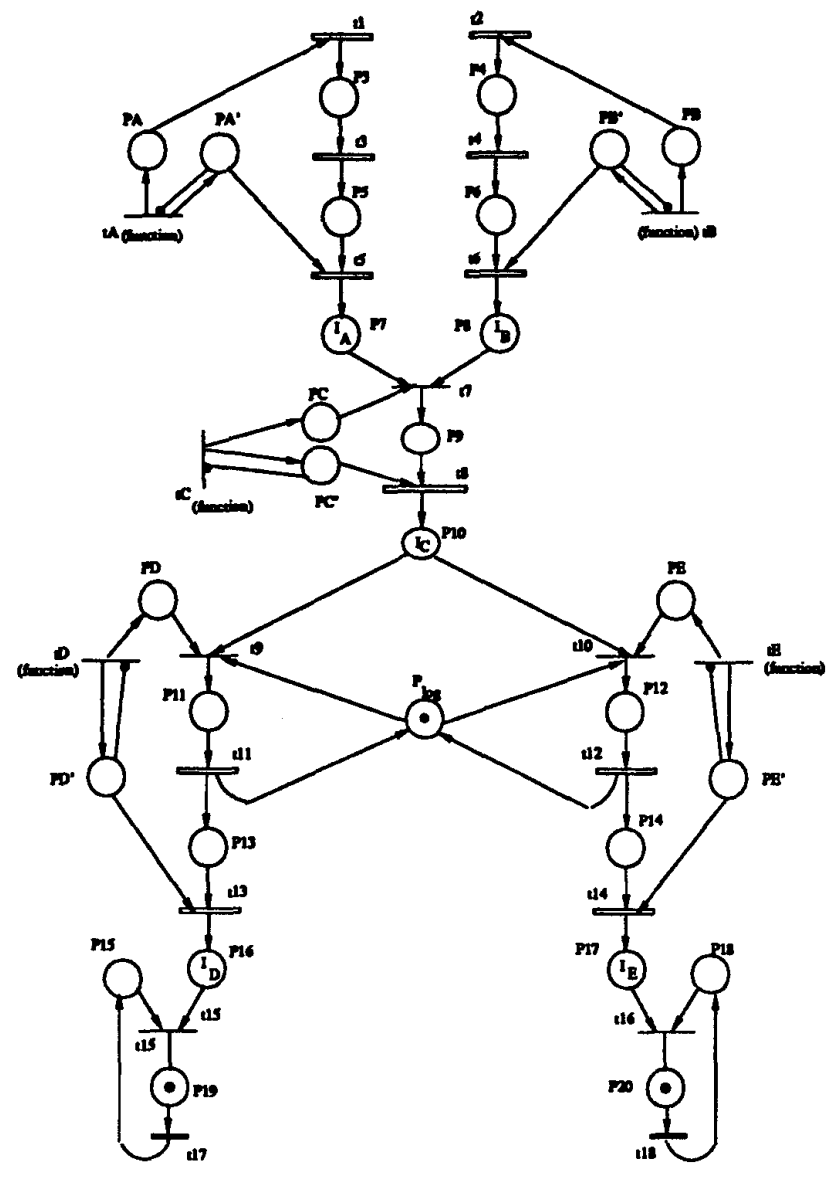

Figure 4: Petri net model for the reorder point inventory control based supply chain

not steady and dense in nature. The assemble-to-order case offers lesser costs for the supply chain in terms of holding inventory, but the downside is customer order lead times. That is to say, if the customers at warehouses $W_{1}$ and $W_{2}$ can not wait until the product is assembled to order, this case won't fit the bill. In the GSPN model for such a system (which is not shown in the paper) the production of $\mathrm{D}$ and $\mathrm{E}$ are triggered directly by customer orders. Hence the places PD, $\mathrm{PD}^{\prime}, \mathrm{PE}$, and $\mathrm{PE}^{\prime}$ found in Figure 4 will be absent. Also transitions $t D$ and $t E$ are absent. The initial marking will not have tokens in places $P 16$ and $P 17$. The performance measures of interest are the average work in process and average finished goods inventories of materials/goods $A$ through E; the lead times for order delivery; and, material replenishment cycle times (or, the supply chain lead times) for D and E. These are coded into the total cost, to be defined below.

\subsection{Numerical Results}

Here, we wish to evaluate the performance of the supply chain in terms of the total cost, which is the sum of the total inventory carrying cost and the cost incurred due to delayed deliveries.

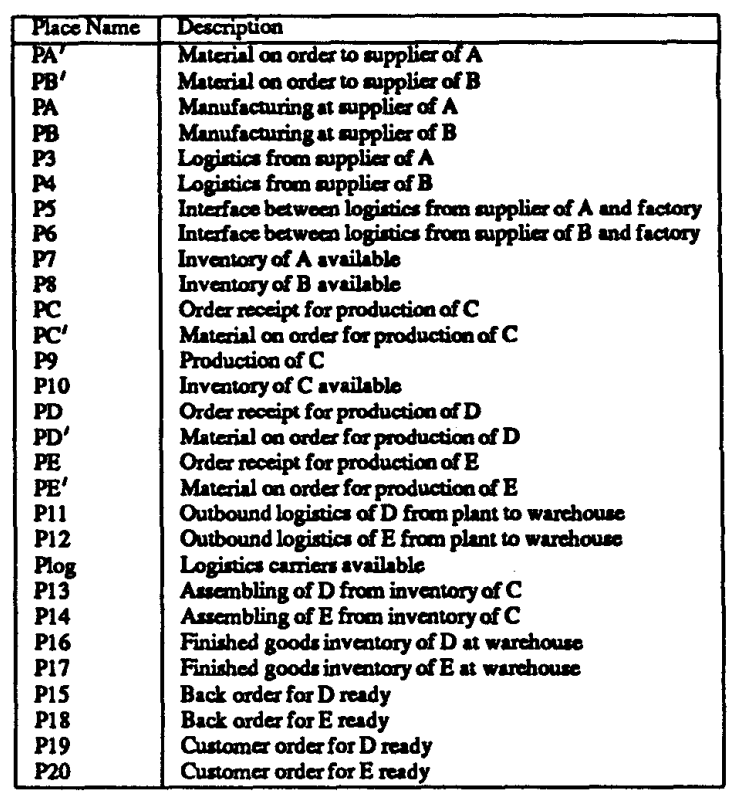

Table 1: Description of the Petri net for reorder point based supply chain

It is clear that the above components of the total cost are complementary to each other. There is a trade-off between the two which is clearly brought out by the two replenishment policies followed, viz. the reorder point based maketo-stock system and the assemble-to-order system. Let the holding cost incurred for inventory be $H_{I}$, and the cost per hour of delayed delivery be $H_{D}$. We varied the ratio of the component costs, $\frac{H_{D}}{H_{l}}$ from 1.5 to 40.0 to observe any trends in the total cost, so that the decision maker can choose the appropriate policy. We assume that product $E$ is valued more than product $\mathrm{D}$ by $50 \%$. That is, delayed deliveries for $\mathrm{E}$ are costlier by $50 \%$ when compared to that of $D$.

The net inventory is computed as follows:

1. For the make-to-stock system, it is the sum of the steady state average finished goods inventories of $A, B, C, D$, and E.

2. For the assemble-to-order system, it is the sum of the steady state average finished goods inventories of $A, B, C$, and the steady state average work in process inventories of $\mathrm{D}$ and $\mathrm{E}$, since the last two are not made to stock.

This net inventory is easily obtained from the GSPN analysis. Also, keeping in view that held inventory becomes expensive as we move from raw materials to finished goods, we increase the holding cost rates from the raw materials to the finished goods. Specifically, the finished good inventory of $C$ is $20 \%$ costlier than that of $A$ and B, and the finished goods inventories of $\mathrm{D}$ and $\mathrm{E}$ both cost $20 \%$ more than that of $\mathrm{C}$. 


\begin{tabular}{|c|c|}
\hline Transition Name & Description \\
\hline A & Start of manufacturing of $A$ \\
\hline $\mathbf{t B}$ & Surt of manufacuring of $B$ \\
\hline $\mathbf{t}$ & Processing by supplier of $\mathbf{A}$ \\
\hline+2 & Processing by supplier of B \\
\hline t3 & Transportation from aupplier of $A$ \\
\hline 14 & Transportation from supplier of B \\
\hline 5 & Paper work or interfaces with supplier of $\mathbf{A}$ \\
\hline 16 & Paper work or interfaces with supplier of B \\
\hline tC & Trigger for production of $C$ \\
\hline $\mathbf{t 7}$ & Manufacturer of $\mathrm{C}$ starts production \\
\hline 18 & Processing of C \\
\hline $\mathbf{W}$ & Trigger for assembling of D \\
\hline $\mathbf{t E}$ & Trigger for assembling of $\mathrm{E}$ \\
\hline 6 & End of assembling of $D$ from $C$ \\
\hline t10 & End of assembling of $E$ from $C$ \\
\hline t11 & Outbound logistics of D \\
\hline 12 & Outbound logistics of $E$ \\
\hline t13 & Assembling of D \\
\hline $\mathbf{1 4}$ & Assembling of $E$ \\
\hline t15 & Customer order for D served \\
\hline t16 & Customer order for E terved \\
\hline $\mathrm{t17}$ & Arrival of order for $D$ \\
\hline $\mathrm{t} 18$ & Arrival of order for $E$ \\
\hline
\end{tabular}

Table 2: Description of the Petri net for reorder point based supply chain, contd.

Effect of Arrival Rates of End Products: The influence of arrival rates of $D$ and $E$ on the performance of the system is shown in Table 3. We observe the following:

1. When the ratio $\frac{H_{D}}{H_{1}}=1.5$, the assemble-to-order system dominates make-to-stock system. This implies that when delayed deliveries are not costly, it is better to have the assemble-to-order system.

The total delay in delivery decreases as the arrival rate increases in the assemble-to-order system. This is a direct consequence of Little's Law which states that Inventory = $\lambda *$ Waiting Time. Here, inventory is to be considered as the constant number of tokens floating in the assemble-toorder portion of the Petri net. On the other hand, the waiting times of the end products in the make-to-stock system are found to increase as their arrival rate increases. This is because the arriving orders for $D$ and $E$ are served from the finished goods stock pile. Hence as the arrival rate is increased, orders will have to wait until the stock pile is non-empty.

2. When the ratio $\frac{H_{D}}{H_{I}}=40.0$, delayed deliveries are expensive. Thus one would expect the assemble-to-order system to perform badly, and so it does, as in Table 3. Interestingly, in the case of the make-to-stock system, the total cost appears to be convex in arrival rate with the minimum between arrival rates of 1.0 and 1.2. Thus, it is more economical for the enterprise to choose the the make-to-stock system when the ratio is 40.0 , especially with the arrival rate managed around 1.0 to 1.2 units/hr.

Effect of Targeted Finished Goods Inventory of C: We studied the effect on the total cost of the targeted finished goods inventory of the common base material (C) for producing the end products $\mathrm{D}$ and $\mathrm{E}$.

\begin{tabular}{|c|c|c|c|c|}
\hline \multirow{3}{*}{$\begin{array}{c}\lambda_{D} \\
\text { units/tr }\end{array}$} & \multicolumn{4}{|c|}{ Total Coat } \\
\cline { 2 - 5 } & \multicolumn{2}{|c|}{$\frac{H_{D}}{H_{I}}=1.5$} & \multicolumn{2}{|c|}{$\frac{H_{P}}{H_{I}}=40.0$} \\
\hline 0.8 & 22.421 & 19.815 & 26.001 & 257.437 \\
\hline 1.0 & 21.237 & 18.610 & 25.818 & 237.559 \\
\hline 1.2 & 20.012 & 17.714 & 25.961 & 224.228 \\
\hline 1.4 & 18.774 & 17.016 & 26.339 & 214.675 \\
\hline
\end{tabular}

Table 3: Performance with varying arrival rates of D; MTS: maketo-stock, ATO: assemble-to-order

\begin{tabular}{|c|c|c|}
\hline \multirow{2}{*}{$F G I_{C}$} & \multicolumn{2}{|c|}{ Total Coat } \\
\cline { 2 - 3 } & $\frac{H^{2}}{H_{I}}=1.5$ & $\frac{H_{D}}{H_{I}}=40.0$ \\
\hline 6 & 1854 & 28.01 \\
\hline 9 & 27.53 & 29.34 \\
\hline 12 & 35533 & 42.175 \\
\hline 15 & 43.403 & 49.929 \\
\hline
\end{tabular}

Table 4: Performance of make-to-stock system with various levels of the targeted finished goods inventory of $\mathrm{C}$

1. In Table 4 we show the results for the make-to-stock system. As we increase the finished goods inventory (FGI) of $\mathrm{C}$, the customer order delays decrease due to increased stock piles (and hence lesser back order times). When the ratio of costs is 1.5, the inventories are taxed more than the delays, and hence we see that there is a $134 \%$ increase in total costs when the finished goods inventory of $\mathrm{C}$ is increased by $150 \%$. On the other hand, when the delays are costlier and consequently inventories are taxed lesser (cost ratio $=40.0$ ), we see that the total cost increases by about $78 \%$ only. This suggests that make-to-stock systems are preferable when delay related costs are substantial when compared to inventory costs. This corroborates available literature (see for instance [4]) on such systems.

2. In Table $\mathbf{5}$ we capture the results for the assemble-toorder system. We see that, as in the case of the make-tostock system, increasing the finished goods inventory level of $\mathrm{C}$ increases the total cost. When the finished goods inventory of $\mathrm{C}$ is 6 , the assemble-to-order system performs better than make-to-stock system, when the cost ratio is 1.5 (shown in first columns of Tables 4-5). Also, in the case of the assemble-to-order system, when the cost ratio is 40.0 , the total cost increases by about $6 \%$ when the base stock levels are increased by $60 \%$ from 5 to 8 . This gives us an indication that the assemble-to-order system is almost immune to the finished goods inventory levels of $\mathrm{C}$ when the cost ratio is high. But this is no incentive in going for such a system, for, though the percentage variation is small, the absolute values of total costs are far higher than the case for the make-to-stock system (shown in the last columns of Tables 4-5.)

\subsection{The Product Postponement Problem}

In this section, we discuss very briefly how an integrated GSPN-queueing network model is used to solve the decoupling point location problem. We consider a supply chain 


\begin{tabular}{|c|c|c|}
\hline \multirow{2}{*}{$F G I_{C}$} & \multicolumn{2}{|c|}{ Tot Cost } \\
\cline { 2 - 3 } & $H_{P}=1.5$ & $\frac{f_{p}}{H_{l}}=40.0$ \\
\hline 5 & 15.64 & 197.40 \\
\hline 6 & 18.37 & 201.52 \\
\hline 7 & 21.07 & 204.87 \\
\hline 8 & 23.73 & 207.92 \\
\hline
\end{tabular}

Table 5: Performance of assemble-to-order system with various levels of the targeted finished goods inventory of $\mathrm{C}$

with a pipeline (tandem) kind of structure. Each upstream facility passes on material to the downstream facility. The end customer demands occur at the retail outlets, which is supplied by a single distribution center. We consider only one type of end product. In such a supply chain, we would like to determine the customer order decoupling point. The decoupling point is where the order is assigned to the customer. In other words, till the decoupling point, all the subassemblies are made to stock. After the decoupling point, the item is assigned to the customer, i.e., made-to-order. The costs considered are: the cost of holding inventory that results due to the make-to-stock part of the network, before the decoupling point $\mathrm{D}$, and, the cost of excess lead time that takes to assemble and deliver the customer order, the decoupling point onwards. We observe that the above problem has a structure that makes it amenable for integrated queueing network-GSPN analysis (see [9], pp. 560-570). Specifically, we proceed as follows. We aggregate the subnets (places and transitions) in the GSPN, other than the node corresponding to decoupling point, by using queveing analysis and replacing transition firing rates by resultant throughput rate obtained from the queueing analysis. The assemble-to-order portion of the system is modeled as flow of Kanbans and is best solved using GSPN analysis. Thus, the aggregated system is solved using a combination of queueing and GSPN analysis techniques. An exhaustive enumeration which is of the order of the number of facilities of the supply chain considered, is then done to arrive at the decoupling point.

Preliminary results that we obtained are encouraging. For instance, the decoupling point moves to the right if holding costs are low, and to the left if delay costs are low. Many authors in current literature solve this problem by resorting to simulation, while ours represents one of the first steps in tackling this problem analytically.

\section{Conclusions}

In this paper, we have investigated dynamic modeling techniques for analyzing the supply chain process based on generalized stochastic Petri nets (GSPN). We showed how GSPNs can be used for computation of lead time for both assemble-to-order and make-to-stock supply chains. Our modeling method accounted the logistics process as also the interface process that exist between any two members of the supply chain. We compared two production planning and control policies, viz. the make-to-stock and the assembleto-order systems and discussed when each one is suited. We also proposed a novel way of solving the product postponement problem.

\section{References}

[1] B. C. Arntzen, G. G. Brown, and T. P. Harrison. Global supply chain management at Digital Equipment Corporation . Interfaces, 25(1):69-93, Jan-Feb 1995.

[2] K. Bhaskaran and Y.T. Leung. Manufacturing supply chain modelling and reengineering. Sadhana - Academy Proceedings in Engineering Sciences, 22(2):165-187, April 1997.

[3] M.A. Cohen and H.L. Lee. Strategic analysis of integrated production distribution systems: Models nad methods. Operations Research, 36(2):216-228, March-April 1988.

[4] D. Connors, C. An, S. Buckley, G. Feigin, A. Levas, N. Nayak, R. Petrakian, and R. Srinivasan. Dynamic modeling of re-engineering supply chains. Technical Report RC19944, IBM Research Center, Almaden, 1995.

[5] M. Ettl, G.E. Feigin, G.Y. Lin, and D.D. Yao. A supply network model with base-stock control and service requirements. Technical Report RC 20473, IBM Research Divison, 1996.

[6] N. R. S. Raghavan and N. Viswanadham. Performance modeling and dynamic scheduling of make-to-order supply chains. In Third International Conference on Agile and Computer Integrated Manufacturing, Renesslaer Polytechnic Institute, October 1998.

[7] J. K. Sankaran and N. R. S. Raghavan. Locating and sizing plants to bottle Propane in South India. Interfaces, 27(6):1-15, Nov-Dec 1997.

[8] U. S. Karmarkar, S. Kekre, and S. Kekre. Multi-item batching heuristics for minimization of queueing delays. $E u$ ropean Journal of Operations Research, 58:99-111, 1992.

[9] N. Viswanadham and Y. Narahari. Performance Modeling of Automated Manufacturing Systems. Prentice Hall, Englewood Cliffs, NJ, 1992.

[10] T. E. Vollman, W. L. Berry, and D. C. Whybark. Manufacturing Planning and Control Systems. The Dow JonesIrwin/APICS Series in Production Management; Fourth Edition 1998. 